\title{
Survey on Knowledge and Awareness about Human Papilloma Virus and Cervical Cancer in Parents of School-Going Children
}

\author{
Sanika J. Kotkar ${ }^{1}$, Priyanka Gokhale ${ }^{2}$ \\ ${ }^{1}$ Student (BPT), DPO's Nett College of Physiotherapy, Thane. \\ ${ }^{2}$ Assistant Professor, DPO's Nett College of Physiotherapy, Thane. \\ Corresponding Author: Sanika J. Kotkar
}

\begin{abstract}
Human papilloma virus lesions are known to cause cervical cancer in persons infected by the virus. The incubation time between an oncogenic HPV infection and cervical cancer is 10-15 years hence preventive measures should be taken in adolescent age. HPV vaccines (Cervarix and Gardasil) reduce the risk of cancerous and precancerous lesion by more than $95 \%$. These vaccines are to be admitted to young children before their sexual debut (11-12 years), since deemed as legal minors, parental consent is required for the administration of the virus. Parental knowledge about cervical cancer and HPV infections plays a vital role in their awareness about the presence of HPV vaccines and their willingness to get their child vaccinated against the same. The study consisted of a pre-validated questionnaire posed before the parents to know their knowledge and awareness regarding cervical cancer, human papilloma virus and sexual health.
\end{abstract}

Keywords: Cervical cancer, Human Papilloma Virus, knowledge, awareness, school children, cancer

\section{INTRODUCTION}

Cancer is defined as a clonal disorder of dysregulated growth and differentiation leading to a persistent accumulation of phenotypically altered cells and tissues, which if left unchecked, will result in death or severe morbidity. ${ }^{[1]}$ Cervical cancer or Cervical Intraepithelial Neoplasia (CIN) refers to the histopathological description in which a part or full thickness of stratified squamous epithelium is replaced by cells showing varying degrees of dysplasia. ${ }^{[2]}$

Human Papilloma Virus (HPV) is a small DNA virus which infects the basal cells in stratified squamous epithelium at the squamo-columnar junction of uterine cervix ${ }^{[3]}$ HPV lesions arise from unchecked cell proliferation and mutations and lead to cancer ultimately. ${ }^{[4]}$ HPV infections are easily transmitted, possibly through microscopic tears in the surface of epithelium occurring during coitus. ${ }^{[3]}$ Though infections of introitus and vagina are as common as cervical infections, they seldom result in cancer. ${ }^{[3]}$ Out of more than 40 anogenital types of HPV, about 15 are carcinogenic and HPV types 16 and 18 cause nearly $70 \%$ of cervical cancer. ${ }^{[3]}$ Persistent infection by high-risk HPV is the main cause of cervical cancer. ${ }^{[5]}$ HPV infection is the most prevalent sexually transmitted disease (STD), which results in over 14 crore individuals annually and $80 \%$ of sexually active individuals in their lifetime to be infected from HPV. ${ }^{[4]}$

According to the World Health Organization (WHO), cervical cancer is the $2^{\text {nd }}$ most common cancer in women living in less developed regions with an estimated 5.7 lakh cases and 3.11 lakh deaths in 2018 and more than $85 \%$ of these deaths occurring in 
less developed regions. ${ }^{[5]}$ Cervical cancer is the $4^{\text {th }}$ cause of cancer death in women. ${ }^{[5]}$ The age-standardized incidence rate (ASIR) for cervical cancer is lower in developed nations at 5.0 per 100,000 compared with developing nations at 8.0 per $100,000 .^{[6]}$ Similarly, the age-standardized death rate (ASDR) for cervical cancer is lower in developed nations at 2.2 per 100,000 compared with developing nations at 4.3 per $100,000{ }^{[6]}$ This cancer is more incident in developing countries, because of less access to prevention, screening and treatment programs. ${ }^{[5]}$ In comparison to developing countries with poor vaccination coverage and lack of organized cervical cancer screening programs, developed countries with well-organized cervical cancer screening programs have gained significant reduction in cervical cancer incidence and mortality. $^{[6]}$

Cervical cancer is the only cancer which is preventable with the help of a vaccine. ${ }^{[7]}$ Surveillance data on worldwide cancer survival shows wide variation between nations, and these data have been used as a metric of the effectiveness of health systems in cancer prevention, control and treatment. ${ }^{[6]}$ It is one of the most preventable and treatable forms of cancer. ${ }^{[7]}$ HPV vaccine prevents cancer caused by HPV infections. ${ }^{[7]}$ Introduction of vaccines was a point of transition in medicine. ${ }^{[7]}$ To reduce the cases of cervical cancer and HPV related diseases, prophylactic HPV vaccines have been developed as a primary prevention strategy. ${ }^{[5]}$ First HPV vaccine was licensed in the USA in 2006 and Centre for Disease Control (CDC) and prevention first recommended routine HPV vaccination for girls aged 11-12 years in 2007. ${ }^{[8]}$

Currently, 2 vaccines are available which reduces the risk of cancerous and precancerous changes of cervix by more than $95 \% .^{[9]} 3$ varieties of the available prophylactic vaccines (bivalent, quadrivalent, nonavalent) vary in number of HPV types they contain and target. ${ }^{[3]}$ These vaccines contain Virus-Like Particles (VLPs) produced by recombinant-DNA technique, which creates L1-protein. ${ }^{[3]}$ Vaccinated persons are protected by VLPs that can stimulate generation of high titres neutralizing antibodies independent of cellmediated immune response. ${ }^{[3]}$ HPV vaccines are considered safe and effective for prevention of HPV infection. ${ }^{[5]}$ These vaccines induce the immune system against HPV 6, 11, 16, 18 and some other types. ${ }^{[9]}$ Primary target population for HPV vaccination is girls before initiation of sexual activity. ${ }^{[7]}$ It is recommended for preteens (both boys and girls) aged 11-12 years but can be given as early as age 9 and until age 26 for females and age 24 for males. ${ }^{[7]}$

Comprehensive cervical cancer control is composed of primary prevention (vaccination against HPV), secondary prevention (screening and treatment of precancerous lesions), tertiary care (diagnosis and treatment of invasive cervical cancer) and palliative care. ${ }^{[4]}$ Early detection of cancerous lesions through preventive strategies and tools leads to a positive prognosis and a higher chance of the patient being fully cured. ${ }^{[10]}$ Evidence showed that education by raising awareness, improving accessibility to services, increasing individuals' efficacy and empowering over future choices can enhance the demand for preventative health services. $^{[11]}$ The world health education mentions that "Health education is an exchange of information with the purpose of increasing awareness and knowledge about how to keep healthy and prevent diseases (such as cervical cancer), including information about resources that are available and the benefits of accessing services" (Health, Diseases, and Promotion, 2006). ${ }^{[11]}$

Due to poor knowledge about screening for HPV infections and cervical cancer, they are diagnosed at a late stage where treatment becomes difficult and puts the woman at a risk of complications from the disease and from the treatment as well. These factors can precipitate comorbidities 
and reduce the quality of life in many women. ${ }^{[11,12,13]}$

Complications following chemo radiotherapy and radical surgical treatment of cervical cancer include: Bowel-bladder dysfunction, lower limb lymphadenopathy, sexual dysfunction, decreased bone mineral density, chronic fatigue, psychosocial problems, and decreased cardiovascular endurance. ${ }^{[11,12,13]}$

Many studies report a diminished quality of life among cervical-cancer survivors due to treatment-related effects. The quality of life is found to be lower than not only the general population, but also lower than people with other pelvic cancers. $[11,12,13]$

Cervical cancer is an important disease that requires attention even at the time of adolescence, keeping prevention in mind. ${ }^{[7]}$

Vaccines are one of the most effective public health intervention protecting against infectious diseases. ${ }^{[7]}$ The success of the vaccine depends upon levels of acceptability and uptake, which heavily rely on willingness of parents to have their eligible daughters vaccinated. ${ }^{[7]}$ Since the vaccine is targeted at adolescents who are regarded as 'legal minors', parental consent for vaccines to be administered is important. $^{[7]}$ Parental literacy has been associated with knowledge of cervical cancer and HPV infection amongst women. ${ }^{[3]}$ There is a very less data available on the knowledge and awareness about cervical cancer and HPV vaccination in parents of younger population in the Indian subcontinent, hence this study was undertaken.

\section{METHOD}

\section{Ethical clearance}

The study was reviewed by the Ethical Committee and the self-made questionnaire was validated for use.

\section{Study design and participants}

A cross-sectional study was conducted in schools and tuitions in the city of Mumbai. The study population consisted of parents of school-going children aged 1215 years. The study was carried out for 18 months.

\section{Data collection}

With the support of school and tuition administrations, Parent Teacher Association representatives and students, the online Google-form based questionnaire was distributed through social media platforms to the parents. The parents were explained about the purpose of the present study and were educated on how to fill the online questionnaire. Assistance was provided to people with language barriers and inaccessibility to smartphones and internet connection. The parents who agreed to take part in the study all signed the informed consent form. The questionnaire was developed considering the aim of the present study and the wordings of the questionnaire were framed such that to ensure understandability and validity of the responses. The questionnaire consisted of open questions that addressed the sociodemographic data and multiple choice questions addressing knowledge about Human Papilloma Virus (HPV), HPV vaccine, cervical cancer and awareness about their children's sexual health. The mean time required to fill the survey was 45 minutes.

\section{Data analysis}

The data was entered in Microsoft Excel spreadsheet (v.2013) and analyzed. Answers to open questions were categorized according to their similarities. Whereas answers to closed end questions was calculated in percentage according to the number of a response of each participant based on total responses.

\section{RESULTS}

\section{Cervical Cancer and Human papilloma Virus:}

Out of the total study population of 1,009 respondents, $50.84 \%$ respondents have heard about cervical cancer while the 
remaining $49.16 \%$ have not heard about cervical cancer. But $70.76 \%$ of the total respondents were not sure whether a HPV infection can cause cervical cancer, $12.19 \%$ think it does not whereas only $17.05 \%$ people are aware that HPV infections can cause cervical cancer. $57.38 \%$ respondent parents think that cervical cancer can be prevented.

When parents were asked if they were aware about the presence of a vaccine against HPV and cervical cancer, $70.76 \%$ are unaware about the presence of a vaccination against HPV infections and cervical cancer while only $29.24 \%$ of the total respondents were aware about the HPV vaccine. $74.23 \%$ people didn't know about the route of administration of the HPV vaccination and only $17.15 \%$ parents voted correctly that the HPV vaccination is administered intramuscularly with the favourable sites for injection being the deltoid, quadriceps and gluteus maximus muscles. $76.91 \%$ of the total parents have not had their child/children vaccinated against HPV and cervical cancer whereas only $23.09 \%$ of the total parents have had their child/children vaccinated against the same. Out of the total study population, only $61.55 \%$ of the parents were willing to vaccinate their child/children against HPV.

\section{Sexual Health:}

Parental awareness about their child's sexual health and how they educate them about it plays a vital role as the HPV vaccinations are given to the children in adolescent age of 9-12 years or before their sexual debut. $83.75 \%$ respondent parents voted that they educate their child/children on matters of sexuality, sexual health and reproduction. Only $33.40 \%$ of the total parents were aware if their child had attained their sexual debut or if he/she was sexually active at present, $38.75 \%$ parents were unaware, while $27.85 \%$ parents were not sure about their knowledge. To educate the child about matters of reproduction and discuss sexuality with them, parents need to be comfortable themselves, $74.23 \%$ parents were comfortable discussing matters of sexuality and reproduction with their child/children and $25.77 \%$ parents replied not. $90.98 \%$ parents agreed that sex education should be taught from an adolescent age as opposed to the remaining $9.02 \%$ parents and the most ideal age for education was voted $12-15$ years.

\section{DISCUSSION}

The study consisted of total 1,009 participants who fit into the inclusion and exclusion criteria of the study. The respondent parents consisted of 551 males and 458 females with a mean parity of 1.49 offspring and 453 respondents having more than 1 offspring. The educational attainment of all the respondents was graduation and above.

\section{Cervical Cancer and Human Papillomavirus}

The study shows that there is a low level of awareness about cervical cancer and HPV vaccine among parents of schoolgoing children. Low level of knowledge can be attributed to the lack of introduction of cervical cancer, HPV infections, HPV vaccinations among the population from their adolescence which carries with them in their adulthood, and later fail to educate their child/children regarding the same or having their child/children vaccinated against HPV infections and cervical cancer.

Out of 1,009 respondents, 50.84\% parents have heard about cervical cancer. But $70.76 \%$ parents are not sure whether a HPV infection can cause cervical cancer. $42.62 \%$ parents are not aware that cervical cancer is preventable. When parents were asked if they were aware about the presence of a vaccine against HPV and cervical cancer, $70.76 \%$ respondent parents were unaware about the same.

Out of the total study population, only $23.09 \%$ parents have gotten their child/children vaccinated and $76.91 \%$ have not till the present date. The reasons for not getting their child/children vaccinated were the parents being unaware about the 
vaccine, their doctor not recommending for the vaccination, no specific reason to get vaccinated, their children not needing the vaccination either because they were boys or did not show any signs of the infection yet, unsure about the safety of the vaccine, high cost of the vaccine and unavailability of the vaccine in their province. Whereas the reasons which led the parents to get their child/children vaccinated against HPV infections and cervical cancer were due to their doctor's orders, by their own decision and research and due to past history of cervical cancer in the family.

$38.45 \%$ of the total respondent parents remained unwilling to get their child/children vaccinated against HPV infections and cervical cancer as they were unaware about the vaccination, they stated that boys do not need any vaccination as they cannot contract cervical cancer, their children hadn't gotten infected yet or they don't need it.

\section{Sexual Health}

The study also points out the presence of social stigma faced by the parents regarding discussing about matters of sexuality or reproduction with their children or being approachable to the children for the same. As the vaccination is supposed to be administered at an age where the children are considered to be legal minors and need their parents' permission for the same, parental education regarding sexuality, reproduction, cervical cancer, HPV vaccination, etc. plays a vital role in prevention of HPV infections and cervical cancer in these children. Since the latent period between a high risk HPV infection and cervical cancer is 10-15 years, prevention and education in the adolescent age is of crucial importance. Due to westernization, children are introduced to the concepts of sexual health and reproduction at an early age where the source of their knowledge is based on social media or their exposure to teen drama series. Both these sources do not display the full picture and leave the children to explore the knowledge regarding sexual health on their own resulting in children attaining their sexual debut at an early age, multiple partners in their life, unwanted pregnancies, substance abuse, hormonal dysfunctions, etc. Hence educating the child regarding the matter is important.

The results of this study may contribute to prompt health promotion actions aimed at improving knowledge about cervical cancer and HPV vaccination, adherence to vaccination and other HPV prevention methods to reduce the incidence of HPV infections and other sexually transmitted diseases, thereby reducing the incidence or timely diagnosis of cancerous and precancerous cervical intraepithelial neoplasias.

\section{CONCLUSION}

On the basis of our analysis, our study concludes that there are low levels of awareness about HPV vaccine and cervical cancer in the parents of school-going children possibly due to poor knowledge regarding HPV infections, route of transmission of the infection, cervical cancer and vaccination against the same.

\section{Acknowledgement: None}

\section{Conflict of Interest: None}

\section{Source of Funding: None}

\section{Ethical Approval: Approved}

\section{REFERENCES}

1. Yash Pal Munjal. API Textbook of Medicine. $9^{\text {th }}$ Edition. Mumbai: The Association of Physicians of India; 2012. 21.2 Principles of Cancer Biology and Pathology, Pg. No. 1561.

2. Howkins \& Bourne. Shaw's Textbook of Gynaecology. $16^{\text {th }}$ Edition. New Delhi, India: Reed Elsevier India Private Limited; 2015. 38. Cervical Intraepithelial Neoplasia, Carcinoma of cervix, Pg. No. 485.

3. Jadhav et al. Awareness of Cervical Cancer and vaccination against HPV among Urban women. European Journal of 
Pharmaceutical and Medical Research. 2020, 7 (12), 380-383.

4. R Wang et al. Human papillomavirus vaccine against cervical cancer: Opportunity and challenge. Cancer Letters. 2020, 471, 88-102.

5. Santos et al. Knowledge about Cervical Cancer and HPV immunization dropout rate among Brazilian adolescent girls and their guardians. BMC Public Health. 2020, 20:301.

6. Musa $\mathrm{J}$ et al. Effect of cervical cancer education and provider recommendation for screening on screening rates: A systematic review and meta-analysis. PLoS ONE 12(9): e0183924 (2017).

7. Haleemat Wuraola Akinleye et al. Parental willingness to vaccinate adolescent daughters against human papilloma virus for cervical cancer prevention in Western Nigeria. Pan African Medical Journal. 2020;36:112.

[doi: 10.11604/pamj.2020.36.112.19007]

8. Landy et al. Impact of screening on cervical cancer incidence: A population-based casecontrol study in the United States. International Journal of Cancer. 2020, 147, 887-896.

9. Mohammad Raza Haghshenas, Tahoora Maousan and Mahmood Moosazadeh. Efficacy of Human Papilloma Virus L1
Protein Vaccines (Cervarix and Gardasil) in Reducing the Risk of Cervical Intraepithelial Neoplasia: A meta-analysis. International Journal of Preventive Medicine. 2017, 8:44.

10. Marques et al. Factors associated with cervical cancer screening participation among migrant women in Europe: A scoping review. International Journal for Equity in Health (2020) 19:160.

11. Marzieh Saei Ghare Naz et al. Educational Interventions for cervical cancer screening behaviour of Women: A systematic review. Asian Pacific Journal of Cancer Prevention, Vol 19 (4), 875-884.

12. Krista S. Pfaendler, Lari Wenzel, Mindy B. Mechanic et. al. Cervical cancer survivorship: Long-term quality of life and social support. Clin Ther. 2015 Jan 1; 37(1): 39-48. doi: 10.1016/j.clinthera.2014.11.013

13. Zhou W et al., Survey on cervical cancer survivors regarding quality of life and sexual function. Journal of Cancer Research and Therapeutics. 2016; 12:938-44.

How to cite this article: Kotkar SJ, Gokhale P. Survey on knowledge and awareness about human papilloma virus and Cervical Cancer in parents of school-going children. Int $J$ Health Sci Res. 2021; 11(9): 27-32. DOI: https://doi. org/10.52403/ijhsr.20210904 